\title{
Recurrent Novae: A Masquerade
}

\author{
Ashley Pagnotta* $\ddagger$ \\ American Museum of Natural History, USA \\ E-mail: ashley.pagnotta@gmail.com
}

Due to low discovery efficiencies, there must be some number of classical novae $(\mathrm{CNe})$ that are in fact recurrent novae ( $\mathrm{RNe}$ ) for which only one eruption has so far been observed. We collect all available information on the light curves, spectra, and quiescent characteristics of a time-limited sample of 237 Galactic CNe and the ten known Galactic RNe, and identify seven characteristics that are indicative of a short recurrence time: (1) outburst amplitude smaller than $14.5-4.5 \times \log \left(t_{3}\right)$, (2) orbital period $>0.6$ days, (3) infrared colors of $J-H>0.7$ mag and $H-K>0.1 \mathrm{mag}$, (4) FWHM of $\mathrm{H} \alpha>2000 \mathrm{~km} \mathrm{~s}^{-1}$, (5) high excitation lines, such as Fe X or He II, near peak, (6) eruption light curves with a plateau, and (7) white dwarf mass greater than $1.2 M_{\odot}$. We use these characteristics to identify good RN candidates from among the list of known $\mathrm{CNe}$ and to draw conclusions about the population of Galactic RNe, specifically related to whether there are enough RNe to provide the observed Type Ia supernova rate, as RNe are good candidate progenitors. We find that $25 \% \pm 10 \%$ of $\mathrm{CNe}$ are likely RNe with only one eruption observed so far, and that RNe could perhaps provide up to one half of the Type Ia supernovae.

The Golden Age of Cataclysmic Variables and Related Objects - III

7-12 September 2015

Palermo, Italy

\footnotetext{
* Speaker.

$\dagger$ This research was funded by the Kathryn W. Davis Postdoctoral Scholar program, which is supported in part by the New York State Education Department and by the National Science Foundation under grant numbers DRL1119444 and DUE-1340006. This conference presentation was supported by the AAS International Travel Grant. We acknowledge with many thanks the variable star observations from the AAVSO International Database contributed by observers worldwide and used in this research.

$\ddagger$ This work is based on Pagnotta \& Schaefer (2014), but includes updates on more recent plate stack searches and collects information on novae that were not included in the original paper but were of interest to other conference attendees.
} 


\section{Introduction \& Motivation}

All novae consist of a white dwarf (WD) accreting material—usually hydrogen - from the outer layers of a nearby companion star onto its surface. The nova eruption itself occurs when the accreted layer reaches a critical temperature and pressure, at which point a runaway nuclear reaction is triggered and the energy from the fusion causes the outer (accreted) layers of the WD to be ejected and the system to dramatically brighten over an average timespan of a few days to weeks. Following the eruption, the accretion in the system resumes and material starts accumulating again on the surface of the WD. Novae are often divided into two general categories: classical novae $(\mathrm{CNe})$, which have only one known eruption, and recurrent novae (RNe), for which multiple eruptions have been observed. The eruption mechanisms in $\mathrm{CNe}$ and $\mathrm{RNe}$ are identical, but two conditions in RNe contribute to the short amount of time needed for enough matter to accumulate to trigger the next nova eruption: the presence of a high mass WD and a high accretion rate, $\dot{M}$ [Pagnotta \& Schaefer(2014)]. These conditions are in fact both necessary to have an RN; with only one, the system will still be a CN. We note that CNe likely do recur eventually, on timescales of $\sim 10^{5}-10^{6}$ years, but for the purpose of this study, we consider a recurrence time of $<100$ years to be what defines an RN.

The number of Galactic novae is poorly known. Although the eruptions are relatively bright, peaking at an absolute magnitude of around $V \approx-8$, a number of factors interfere with our observations of novae, leading to low average discovery efficiencies. Over the past $\sim 125$ years during which we have been systematically recording our observations of the night sky, the coverage, sensitivity, and frequency of these observations has varied widely. Early observations were made with photographic plates, led by the Harvard College Observatory based in Cambridge, MA, USA, and the Sternwarte Sonneberg (Sonneberg Observatory) based in Sonneberg, Thüringen, Germany. Most survey plates are only sensitive to a magnitude of $B \approx 14$, and a number of novae have peak apparent magnitudes fainter than this. Modern surveys with CCD cameras can see to much fainter magnitudes, but end up missing novae that peak brighter than $B \approx 5$ because they are too bright for the camera. No type of survey can detect novae when they are near solar conjunction (all known novae except for one (V959 Mon) have been originally discovered in optical, although many were then observed at other wavelengths), and most surveys, especially the photographic plate programs that dominated the majority of the 20th century, avoid observing near the full moon. Combined, the sun and moon effects can reduce the number of days a nova is detectable in a survey by more than half of the days in the year. The usual observing woes of clouds, bad seeing, and mechanical problems have a negative effect on nova discovery efficiencies as well.

[Shafter(2002)] showed that novae of average brightness $\left(m_{\mathrm{v}, \max } \approx 8\right)$ are discovered only $\sim 10 \%$ of the time, even under the idealistic assumption that $100 \%$ of all very bright novae are discovered [Shafter(2002)]. To be properly classified as recurrent, at least two eruptions of an RN must be observed, with both the first and second eruptions usually being detected via random, undirected searches. The $\sim 10 \%$ discovery figure therefore applies to both eruptions in general, meaning that a large number of eruptions are missed, and a smaller number of systems are seen to have only one eruption and miscategorized as $\mathrm{CNe}$. This is illustrated schematically in Figure 1. The entire largest square represents the full set of Galactic RNe. The smaller white square represents the systems for which no eruptions are seen, the light blue rectangles represent systems 
for which only one eruption is seen, and the small dark blue square represents the systems for which two eruptions are observed and the system is recognized as an RN. As shown in the Figure, this last category is clearly a very small percentage of the overall total number of existing $\mathrm{RN}$ systems.

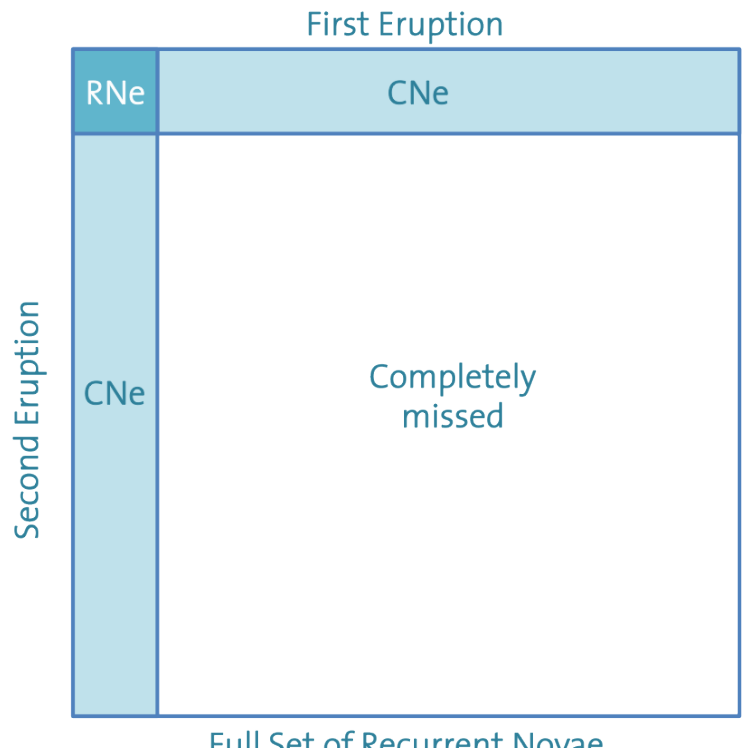

Figure 1: This schematic illustration of the full set of RNe in our Galaxy (represented by the largest square) shows in a general way how few of the RNe are actually recognized to be recurrent. There is a low discovery efficiency for novae due to a number of factors discussed in Section 1 , with only $\sim 10 \%$ of eruptions being discovered on average [Shafter(2002)]. The light blue horizontal bar across the top of the schematic represents the systems for which a first eruption is seen during an initial (undirected) search. The light blue vertical bar across the left side represents the systems for which a second eruption is observed, again during an undirected search. (With rare exception, the second eruption of an RN is discovered as randomly as the first.) The overlap of these two light blue bars, the colored dark blue square on the figure above, represents the systems for which both eruptions are actually observed and are therefore properly labeled as RNe. The white expanse represents the large number of RNe in our Galaxy for which we never actually observe any eruptions.

In reality, the situation is of course more complicated, with nova discovery efficiencies varying from year to year as well as system to system. Additionally, the chance of finding an eruption depends heavily on whether a directed or undirected search is made. Most RN eruptions are found during undirected searches, in which an eruption of that given system is not being sought, but instead observers are looking for transients in general or some completely unrelated science goal. In three cases, however, a directed search has been used to discover either the second eruption of an RN candidate, confirming its RN status. In the directed cases, the discovery efficiency is much higher, because that particular system is being watched closely, although still not perfect, since eruptions can be missed during opposition, full moon, or a spate of bad weather.

One of the main reasons we are interested in the number of Galactic $\mathrm{RNe}$ is to determine whether there are enough to account for the observed rate of Type Ia Supernovae (SNe Ia). SNe Ia are well known for their utility as cosmological distance markers, but the identity of the progenitors is still poorly constrained. Although we have good evidence that there are multiple progenitor chan- 
nels [Brandt et al.(2010), Greggio(2010)], we seek to better understand the progenitor distribution and its effect on the cosmological measurements.

\section{Characteristics of Recurrent Novae}

We identify seven characteristics of novae that are indicative of a short recurrence time, two of which are new. We also discuss an eighth characteristic that may be linked to RNe but is not well-enough understood at this time. All seven of our primary characteristics can be linked to at least one of the two required conditions for RNe discussed in Section 1: a high accretion rate in the system or the presence of a high-mass WD. Although many of these characteristics have previously been used on their own as being indicative of $\mathrm{RNe}$, a single good feature is not enough to give any true insight into the status of the system, especially if there are other contradictory indicators. The seven characteristics we consider are (1) location on a plot of eruption amplitude vs. decline time, (2) the orbital period $\left(P_{\text {orb }}\right)$ of the system, (3) infrared (IR) colors that indicate the presence of a red giant (RG) secondary, (4) a high expansion velocity during outburst, measured by the full-width half-max (FWHM) of the $\mathrm{H} \alpha$ line near peak, (5) the presence of high excitation lines such as $\mathrm{He}$ II or Fe X (or higher) early in the outburst, (6) a plateau in the optical light curve, and (7) a high WD mass measurement. Additionally, we note that the presence of triple-peaked Balmer emission lines early in the eruption may be correlated with a short recurrence time, however the association is still very preliminary.

Throughout the statistical discussion in this section, as well as on Figures 2 and 4, V2487 Oph (Nova Oph 1998) is kept apart from both the CNe and RNe. Using the procedures outlined here we identified it as a likely $\mathrm{RN}$, searched the plate stacks, and found a previous eruption that was observed in 1900 [Pagnotta et al.(2009)]. Because of this, we do not consider V2487 Oph when doing the statistics, to keep them properly independent. Additionally, for each characteristic, we calculate the statistics based on the number of novae for which that characteristic has been measured and reported, not the entire set of $\mathrm{CNe}$, because many of them are poorly observed.

We can asses the strength of each indicator by looking at the number or percentage of $\mathrm{CNe}$ that fall in the $\mathrm{RN}$ range. Classification characteristics with a large number of $\mathrm{CNe}$ in the $\mathrm{RN}$ region are weak indicators, whereas characteristics with only a few "misplaced" $\mathrm{CNe}$ are likely much stronger indicators. For example, we can compare the amplitude vs. decline time statistics to those of the high expansion velocity (FWHM of $\mathrm{H} \alpha$ ) numbers. On our version of the amplitude vs. decline time plot (seen in Figure 2 and described in more detail below), we see that there are only three interlopers in the "region devoid of classical novae", i.e. the systems with $A-A_{0}<0$, and $128 \mathrm{CNe}$ outside of that region (plus the two oddball RNe, T Pyx and IM Nor), where $A$ is the amplitude of the eruption and $A_{0}$ is an empirical threshold line that separates the $\mathrm{RNe}$ from the $\mathrm{CNe}$. If all of the systems with $A-A_{0}<0$ are truly recurrent, then $3 / 131=0.023=2 \%$ of the CNe are actually RNe. Considering instead the FWHM of $\mathrm{H} \alpha$, we see that 30 out of 57 (or 53\%) have a FWHM greater than our initial RN cutoff, which makes for a relatively weak indicator. Because of this, we use a different threshold to identify the good RN candidates, as described in more detail below.

[Duerbeck(1987)] was the first to consider multiple characteristics at once, constructing a plot of amplitude ( $A$ ) vs. time (in days) to decline by 3 mag from peak $\left(t_{3}\right)$ and identifying a "region devoid of classical novae" in the low A, low $t_{3}$ quadrant of the plot. We quantified the Duerbeck 
relation by drawing an empirical threshold $\left(A_{0}\right)$ line on the $A$ vs. $t_{3}$ plot at $A_{0}=14.5-4.5 \times \log t_{3}$, as seen in Figure 2. All of the RNe except T Pyx and IM Nor lie in the region where $A<A_{0}$, or $A-A_{0}=0$. (As T Pyx and IM Nor are unusual systems among the rest of the RNe, it is not unusual to find them outside the "normal" RN region.) Three CNe—LS And, DE Cir, and V1187 Sco-have $A<A_{0}$, for $2 \%$ of the $\mathrm{CNe}$ in the RN region, whereas $78 \%$ of the RNe have $A<A_{0}$. These two fractions differ at the $5.4 \sigma$ level, and, as discussed above, this is a very strong indicator since so few $\mathrm{CNe}$ are mixed in with the $\mathrm{RNe}$.

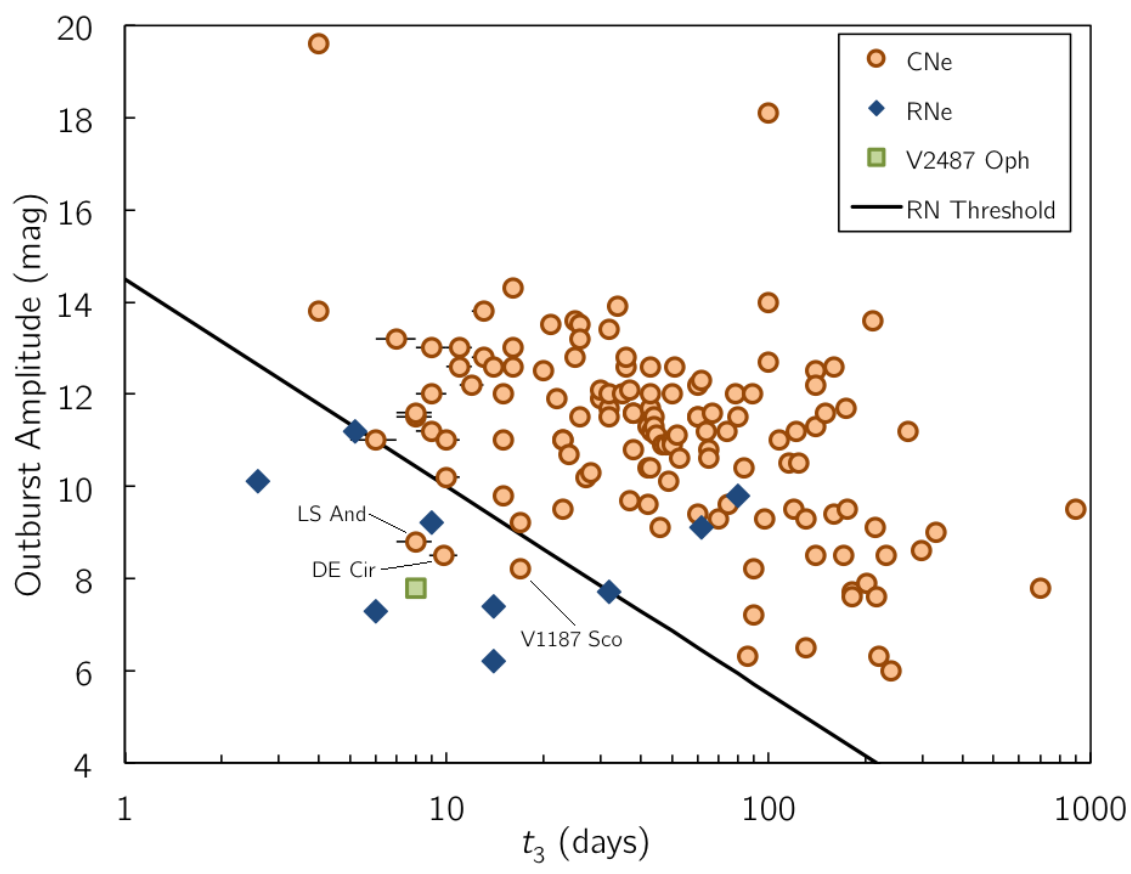

Figure 2: This relationship, plotting the amplitude of the nova eruption vs. $t_{3}$, the time (in days) to decline by 3 mag from peak, was first introduced by [Duerbeck(1987)]. The RNe (dark blue diamonds) are clustered in the lower left quadrant of the figure (with the exception of known oddballs T Pyx and IM Nor) whereas the $\mathrm{CNe}$ are located for the most part in the other three quadrants. The threshold line is empirical, and marks the edge of the RN-region of the plot. Three CNe_-LS And, DE Cir, and V1187 Sco_-are located among the RNe, which indicates they are likely in fact recurrent, not classical. V2487 Oph is also located in the $\mathrm{RN}$ region of this plot, which is one of the characteristics that led us to search for-and find-a previous eruption of this system [Pagnotta et al.(2009)]. Figure originally published in [Pagnotta \& Schaefer(2014)].

The simplest way to drive the high $\dot{M}$ needed for a short $t_{\mathrm{rec}}$ is with the presence of an evolved companion star, usually a subgiant or RG. These evolved companions will have a large radius, $R_{\text {donor }}$, and from Roche Lobe geometry, we know that $P_{\text {orb }} \propto R_{\text {donor }}^{1.5}$, so these systems will have relatively long orbital periods. Looking at the known RNe with measured orbital periods, we see that $78 \%$ (seven of nine) have $P_{\text {orb }}>0.6$ days. Among the $\mathrm{CNe}, 21 \%$ (13 of 62) have periods longer than 0.3 days, and only $13 \%$ ( 8 or 62 ) have $P_{\text {orb }}>0.6$ days. These fractions are different at the $4.5 \sigma$ level.

In addition to long orbital periods, RG companion stars will be visible as an excess in the IR colors of the system. We plot the $J-H$ vs. $H-K$ colors in Figure 3 and, following Figure 1 of 
[Harrison(1992)], we define the RG region to be the stars in the region with $J-H>0.7$ mag and $H-K>0.1$ mag. $15 \%$ (11 of 72) of the CNe have colors that indicate the presence of a RG. $44 \%$ (4 of 9) of the RNe have RG companions. These fractions are relatively similar, but just different enough to be significant (at the $1.7 \sigma$ level). Although the presence of a RG secondary is a good indicator of a possible $\mathrm{RN}$, the lack of an RG does not indicate that the system is a $\mathrm{CN}$.

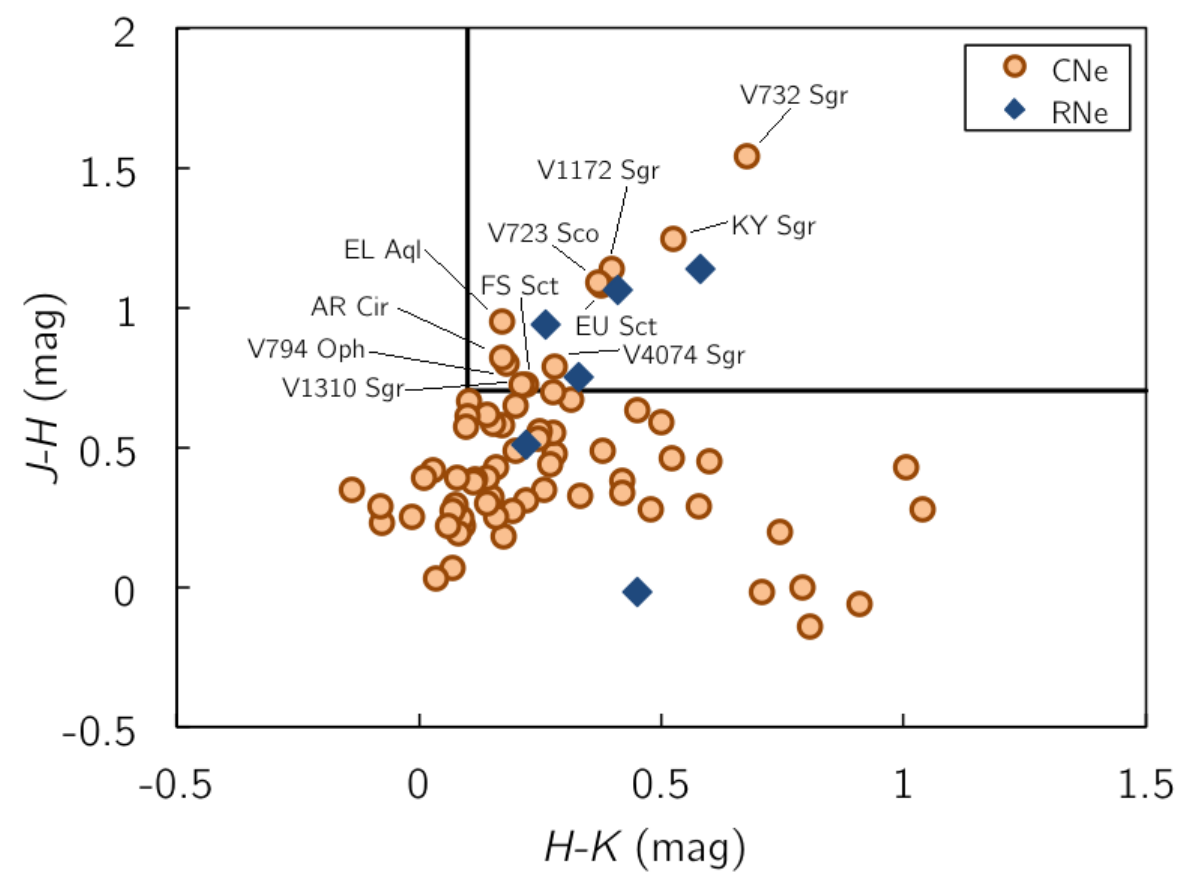

Figure 3: This near-IR color-color diagram allows for the identification of nova systems with RG companion stars, which have a high $\dot{M}$ and therefore a more likely chance of the system being an RN. Four of the known RNe have RG secondaries (T CrB, RS Oph, V3890 Sgr, and V745 Sco) and so although the presence of an RG makes a system more likely to be recurrent, the absence of one is not a strong indicator that the system is truly a CN. The RG threshold $(J-H>0.7$ and $H-K>0.1)$ is based on Figure 1 of [Harrison(1992)]. Figure originally published in [Pagnotta \& Schaefer(2014)].

One of the two new indicators we introduced in [Pagnotta \& Schaefer(2014)] is a high expansion velocity during outburst, as measured by the FWHM of the $\mathrm{H} \alpha$ line near peak. The $\mathrm{H} \alpha$ line is ubiquitous in novae, and the FWHM is both relatively straightforward to measure and commonly reported by observers, making it a useful characteristic. All of the RNe show $\mathrm{H} \alpha$ FWHMs $>2000$ $\mathrm{km} \mathrm{s}^{-1}$, and many are significantly higher, in the 5000-10,000 $\mathrm{km} \mathrm{s}^{-1}$ range. 53\% (30 of 57) of $\mathrm{CNe}$ have $\mathrm{H} \alpha \mathrm{FWHMs}>2000 \mathrm{~km} \mathrm{~s}^{-1}$, and only $14 \%$ (8 of 57) have FWHMs $>3500 \mathrm{~km} \mathrm{~s}^{-1}$. Our interpretation is that novae with $\mathrm{H} \alpha$ FWHMs $<2000 \mathrm{~km} \mathrm{~s}^{-1}$ are likely CNe, while systems with FWHMs $>3500 \mathrm{~km} \mathrm{~s}^{-1}$ are likely RNe. In both the $>2000 \mathrm{~km} \mathrm{~s}^{-1}$ and $>3500 \mathrm{~km} \mathrm{~s}^{-1}$ cases, the differences from the RN population are significant at greater than the $3 \sigma$ level. Figure 4 shows an overlapping histogram of expansion velocities of the $\mathrm{CNe}$ and $\mathrm{RNe}$, in which the difference between the two populations can clearly be seen.

Our second new indicator from [Pagnotta \& Schaefer(2014)] is the presence of unusually high excitation lines in the early outburst spectra, caused by the large amounts of energy needed to 


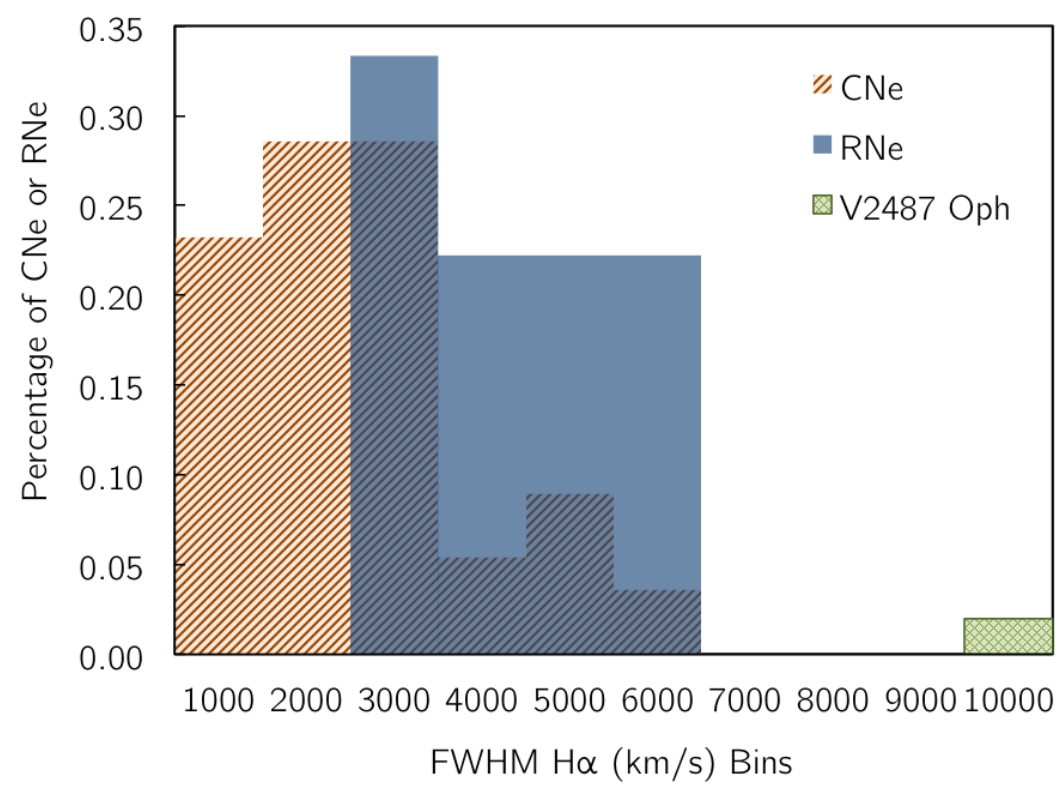

Figure 4: The distributions of the expansion velocities of $\mathrm{CN}$ and $\mathrm{RN}$ eruptions are plotted in this overlapping histogram, with $\mathrm{CNe}$ represented by the brown hashed region and RNe the light blue solid region. As in Figure 2, V2487 Oph is denoted with a different color and pattern. The bins are in steps of $1000 \mathrm{~km} \mathrm{~s}^{-1}$ and are labeled with the (inclusive) maximum value for that bin; the height of each bar shows the percentage of $\mathrm{CNe}$ or $\mathrm{RNe}$ that are located within that bin, where the percentage is calculated using the total number of novae with measured and published early expansion velocities, not the total number of all novae. Figure originally published in [Pagnotta \& Schaefer(2014)].

propel the ejecta out of the deep gravitational well of the high-mass WD. Specifically, we looked for reports of He II near peak and/or iron lines of Fe X or greater. For He II especially it is important to consider only spectra taken near peak, because if the spectra are taken too late then the He II lines may be due to normal nebular emission and not the outburst. All of the RNe show early He II, and 63\% (5 of 8) show high iron. In our sample of novae with reliable spectra where the lines in question were actually sought, 26\% (13 of 50) show He II and 6\% (4 of 64) show Fe X or higher. Only one system, CP Pup, shows both early He II and Fe X or higher. In total, there are 77 systems for which at least one spectral indicator was sought, and 16 of those show early $\mathrm{He}$ II and/or high iron lines, for a total of $21 \%$ that meet this criterion. This is significantly different from the $100 \%$ seen in the RNe.

The sixth indicator we consider is the presence of a plateau in the optical light curve. Light curve morphologies were extensively analyzed by [Strope et al.(2010)], who looked at 77 wellobserved nova eruptions and defined seven light curve classes based on their sample. The largest percentage of novae are classified as S class, with smooth light curves, and significantly fewer in the other classes. Only $17 \%$ of $\mathrm{CNe}$ show plateaus in their light curves and are thus categorized as P class. Depending somewhat on interpretation of sparse light curves, $56 \%-89 \%$ of $\mathrm{RNe}$ have plateaus, with the rest being $\mathrm{S}$ class, and only one of those $(\mathrm{T} \mathrm{CrB})$ being solidly $\mathrm{S}$ with no hint of a plateau. [Hachisu et al.(2008b)] theorize that the plateau is a result of the high-mass 
WD, surrounded by a correspondingly large accretion disk, which reprocesses X-ray light from the nuclear-burning on the surface of the WD into extra optical light, causing the observed plateau. Although the lack of a plateau is not necessarily strong evidence that a system is classical, the existence of one is a strong indicator of a short $t_{\mathrm{rec}}$, with the differences between populations significant at the $8.8 \sigma$ level.

Since a high-mass WD is a necessary condition for an RN, a direct WD mass measurement showing $M_{\mathrm{WD}} \geq 1.2 M_{\odot}$ is an indicator that the system might be recurrent, where the threshold for "high mass" comes from the models of [Yaron et al.(2005)]. Mass measurements of astronomical objects are notoriously difficult and highly uncertain, but are still worth considering. Most of our mass measurements come from a series of light curve model papers by I. Hachisu and M. Kato [Hachisu \& Kato(2004), Hachisu \& Kato(2005), Hachisu \& Kato(2006), Hachisu \& Kato(2007), Hachisu et al.(2008b), Hachisu et al.(2008a), Hachisu \& Kato(2009), Hachisu \& Kato(2010), Kato \& Hachisưu(2003), Kato \& Hachisu(2007), Kato et al.(2009), Kato \& Hachisu(2011)]. In our time-limited sample, we have $27 \mathrm{CNe}$ with $M_{\mathrm{WD}}$ measurements, and of those 27, seven have $M_{\mathrm{WD}} \geq 1.2 M_{\odot}$, for $26 \%$. Although this in and of itself is not enough to cause an RN, these systems are nonetheless good candidates.

Looking at all of the characteristics together for each nova, we can identify systems with multiple RN indicators as good RN candidates. From our compilation of data on our time-limited sample of 237 novae, plus a few more recent novae with characteristics indicating a short $t_{\text {rec }}$ to produce the following list of good candidates, the details of which can be found in Table 4 of [Pagnotta \& Schaefer(2014)]: V1721 Aql, DE Cir, CP Cru, KT Eri, V838 Her, V2672 Oph, V4160 Sgr, V4643 Sgr, V4739 Sgr, and V477 Sct.

\section{Recurrent Nova Fraction}

The $\mathrm{RN}$ fraction $\left(F_{\mathrm{RN}}\right)$ is the percentage of systems that are currently classified as $\mathrm{CNe}$ but are actually RNe for which we have seen one eruption but missed the others for whatever reason. It is not necessarily the fraction of the entire nova population that is recurrent, just the ones which have had only one eruption discovered so far. We can calculate $F_{\mathrm{RN}}$ using three independent methods, which are described in detail in [Pagnotta \& Schaefer(2014)] and which I will briefly review here.

First, we can look at the properties of the $\mathrm{CNe}$, which we have described in previous sections. Using a time-limited sample [Downes et al.(2001)] and the characteristics described above we can evaluate the likelihood of any given $\mathrm{CN}$ in our sample being actually recurrent. Systems with many indications of a short $t_{\mathrm{rec}}$ and no counter-indicators are very likely to be $\mathrm{RNe}$, whereas on the opposite end of the spectrum a system with no indications of a short $t_{\mathrm{rec}}$ or with one or more strong counter-indicators is very likely to be a $\mathrm{CN}$. We placed each system into one of six categories, labeled A-F, where A systems are known RNe, B systems are probably RNe, C systems are likely $\mathrm{RNe}$ but without quite such a strong case, D systems are likely $\mathrm{CNe}$, E systems are certainly CNe, and $\mathrm{F}$ systems do not have enough information known about them to categorize. From the number of systems in each of these categories, we can then calculate $F_{\mathrm{RN}}=(B+C) /(B+C+D+E)$ with uncertainty $\sqrt{F_{\mathrm{RN}} \times\left(1-F_{\mathrm{RN}}\right) /(B+C+D+E)}$. Although this method is subjective, we found that multiple researchers familiar with nova observations independently placed most systems in the same categories, and differences were easily absolved by going back to the original observational 
data. Using this method, we find $F_{\mathrm{RN}}=24.3 \% \pm 3.5 \%$ for the [Downes et al.(2001)] sample. Using a second time-limited sample of just well-observed novae from [Strope et al.(2010)] we get $F_{\mathrm{RN}}=$ $24.1 \% \pm 4.8 \%$, so we take $24 \% \pm 4 \%$ as the final average from this method.

Second, we can deduce $F_{\mathrm{RN}}$ from the number of known RNe, under the reasonable assumption that there must be some proportionality between the number of identified $\mathrm{RNe}$ and the number masquerading as $\mathrm{CNe}$. To understand this proportionality, we take advantage of our thorough understanding of the discovery efficiency of nova eruptions [Pagnotta et al.(2009), Schaefer(2010)]. Using a combination of experience with the plate stack archives and comprehensive records of both amateur and professional nova searches, we can write a relatively simple formula for the discovery efficiency of a nova search: $F_{\text {disc }}=f_{\text {disc }}\left(V_{\text {peak }}\right) \times 0.67 \times\left(t_{3} / 44\right) . f_{\text {disc }}\left(V_{\text {peak }}\right)$ is a discovery efficiency factor that depends on the peak $V$-band magnitude of the eruption, with values ranging from 1.0 for $V_{\text {peak }}=2$ mag or brighter to 0.09 for $V_{\text {peak }}=10$ mag. With this model for discovery efficiency, we run a Monte Carlo simulation with only one free parameter, $F_{\mathrm{RN}}$, which simulates how many nova eruptions will be detected from the known set of $\mathrm{RNe}$ and $\mathrm{CNe}$, as well as how many $\mathrm{RNe}$ will actually have two eruptions detected so that they are properly classified. We vary the input $F_{\mathrm{RN}}$ until the simulation reproduces the observations of exactly ten Galactic RNe and from that we obtain $F_{\mathrm{RN}}=12 \% \pm 3 \%$.

Third, we can look specifically at the discovery efficiencies of the known $\mathrm{RNe}$ and, given how difficult (or not) it is to observe them, deduce how many must be out there for us to have seen the one we know. For example, since T Pyx has a relatively high discovery efficiency and is thus easier to find and recognize as an RN, there do not need to be as many $\mathrm{T}$ Pyx-like systems masquerading as CNe. In contrast, V3890 Sgr has a much lower discovery efficiency, so we deduce that there should be more hidden V3890 Sgr-like systems in existence for us to have detected one of them. We run another Monte Carlo simulation for each of the ten known Galactic RNe to obtain actual numbers, and find that $F_{\mathrm{RN}}=35 \% \pm 3 \%$, but with the caveat that we do not fully understand all of the systematic uncertainties and so the error bar on that calculation is probably much larger than $3 \%$.

In all three of these methods there are undoubtedly systematic errors that we are unaware of and therefore not accounting for, so although the three $F_{\mathrm{RN}}$ values seem disparate, they are likely all within error bars of each other. To account for this, we give our final $F_{\mathrm{RN}}$ value as the straight average of the three values with large error bars, $F_{\mathrm{RN}}=25 \% \pm 10 \%$, or as a range, from $15 \%$ to $35 \%$. Although this is highly uncertain, it is clear that a significant percentage of the listed CNe are in fact hidden RNe. Additionally, our second simulation allows us to estimate how many total active $\mathrm{RNe}$ there are in our Galaxy, which is $\sim 1600 \mathrm{RNe}$, of which we have seen multiple eruptions of 10 systems, and a single eruption of $\sim 100$ systems.

\section{Discussion \& Implications}

Our estimate of $\sim 1600$ Galactic $\mathrm{RNe}$ can be used to determine whether there are enough $\mathrm{RNe}$ to provide the observed Type Ia supernova rate. With the reasonable assumption that RN lifetimes are $\sim(1-2) \times 10^{6} \mathrm{yr}$, we follow the notation and procedure of [della Valle \& Livio(1996)] to obtain an SN Ia birthrate of $(0.8-1.6) \times 10^{-3}$, which is approximately one-quarter to one-half of 
the observed Galactic SN Ia rate. RNe, therefore, can perhaps contribute a significant fraction of the observed Type Ia SNe.

A crucial implication of this work is that the set of $\mathrm{CNe}$ is-and likely always will be-"contaminated" by unrecognized RNe, currently on the order of $\sim 25 \%$ of the set. This should be taken into account when constructing theories and models about novae, since the properties of $\mathrm{RNe}$ can be so strikingly different from those of the $\mathrm{CNe}$. In conjunction with this, we have also been working to find previous eruptions of our best RN candidates by searching archival plates at $\mathrm{HCO}$ and Sonneberg. Table 7 of [Pagnotta \& Schaefer(2014)] lists the details of our searches at the time of that paper's publication. Since then, we have started to check one additional candidate, DE Cir, at HCO. The following plate series have been searched, with no previous eruptions detected: RH/RB 15h, $-60^{\circ}$; RH/RB 16.5h, $-60^{\circ}$; RH/RB 16h, $-75^{\circ}$; RH/RB 14h, $-75^{\circ}$; AM/AC/AX/AY 15h, $-60^{\circ}$; AM/AC/AXY/AY 13.5h, $-60^{\circ}$; AM/AC/AX/AY 16h, $-75^{\circ}$; AM/AC/AX/AY 14h, $-75^{\circ}$; DSY/DSR/DSB $14 \mathrm{~h},-60^{\circ}$.

A significant fraction of the currently-listed $\mathrm{CNe}$ must in fact be masquerading $\mathrm{RNe}$. We should endeavor to unmask these systems to better understand the demographics of the RNe, as well as the theory of novae in general, and their possible connection to SNe Ia.

\section{References}

[Brandt et al.(2010)] Brandt, T. D., Tojeiro, R., Aubourg, É., Heavens, A., Jimenez, R., \& Strauss, M. A. 2010, The Astronomical Journal, 140, 804

[della Valle \& Livio(1996)] della Valle, M., \& Livio, M. 1996, The Astrophysical Journal, 473, 240

[Downes et al.(2001)] Downes, R. A., Webbink, R. F., Shara, M. M., Ritter, H., Kolb, U., \& Duerbeck, H. W. 2001, Publications of the Astronomical Society of the Pacific, 113, 764

[Duerbeck(1987)] Duerbeck, H. W. 1987, in RS Ophiuchi (1985) and the Recurrent Nova Phenomenon, ed. M. F. Bode, 99

[Greggio(2010)] Greggio, L. 2010, Monthly Notices of the Royal Astronomical Society, 406, 22

[Hachisu \& Kato(2004)] Hachisu, I., \& Kato, M. 2004, The Astrophysical Journal Letters, 612, L57

[Hachisu \& Kato(2005)] —. 2005, The Astrophysical Journal, 631, 1094

[Hachisu \& Kato(2006)] —. 2006, The Astrophysical Journal Supplement, 167, 59

[Hachisu \& Kato(2007)] —. 2007, The Astrophysical Journal, 662, 552

[Hachisu \& Kato(2009)] —. 2009, The Astrophysical Journal Letters, 694, L103

[Hachisu \& Kato(2010)] —. 2010, The Astrophysical Journal, 709, 680

[Hachisu et al.(2008a)] Hachisu, I., Kato, M., \& Cassatella, A. 2008a, The Astrophysical Journal, 687, 1236

[Hachisu et al.(2008b)] Hachisu, I., et al. 2008b, in Astronomical Society of the Pacific Conference Series, Vol. 401, Astronomical Society of the Pacific Conference Series, ed. A. Evans, M. F. Bode, T. J. O’Brien, \& M. J. Darnley, 206

[Harrison(1992)] Harrison, T. E. 1992, Monthly Notices of the Royal Astronomical Society, 259, 17P

[Kato \& Hachisu(2003)] Kato, M., \& Hachisu, I. 2003, The Astrophysical Journal Letters, 598, L107 
[Kato \& Hachisu(2007)] —. 2007, The Astrophysical Journal, 657, 1004

[Kato \& Hachisu(2011)] —. 2011, The Astrophysical Journal, 743, 157

[Kato et al.(2009)] Kato, M., Hachisu, I., \& Cassatella, A. 2009, The Astrophysical Journal, 704, 1676

[Pagnotta \& Schaefer(2014)] Pagnotta, A., \& Schaefer, B. E. 2014, The Astrophysical Journal, 788, 164

[Pagnotta et al.(2009)] Pagnotta, A., Schaefer, B. E., Xiao, L., Collazzi, A. C., \& Kroll, P. 2009, The Astronomical Journal, 138, 1230

[Schaefer(2010)] Schaefer, B. E. 2010, The Astrophysical Journal Supplement, 187, 275

[Shafter(2002)] Shafter, A. W. 2002, in American Institute of Physics Conference Series, Vol. 637, Classical Nova Explosions, ed. M. Hernanz \& J. José, 462

[Strope et al.(2010)] Strope, R. J., Schaefer, B. E., \& Henden, A. A. 2010, The Astronomical Journal, 140, 34

[Yaron et al.(2005)] Yaron, O., Prialnik, D., Shara, M. M., \& Kovetz, A. 2005, The Astrophysical Journal, 623,398

\section{DISCUSSION}

JOANNA MIKOLAJEWSKI: Where do the two very fast symbiotic novae (V407 Cyg and Nova Sco 2015) fall on your amplitude vs. $t_{3}$ diagram?

ASHLEY PAGNOTTA: I don't know off the top of my head, but we can definitely look them up and put them on there.

For these proceedings, I used the AAVSO light curves for V407 Cyg and Nova Sco 2015 (V1585 Sco) to measure the amplitude and $t_{3}$ of both eruptions. For V407 Cyg, $A=6.6 \mathrm{mag}, t_{3}=$ 47 days, and $A-A_{0}=-0.4$. For Nova Sco 2015, $A=5.5 \mathrm{mag}, t_{3}=19$ days, and $A-A_{0}=-3.3$. Note that for both novae, the peak brightnesses are not well defined, as we do not have complete light curve coverage, however under the assumption that they were discovered somewhat near peak, both novae are solidly in the RN region of the $A$ vs. $t_{3}$ plot, marking them as potentially good RN candidates. 